\title{
THE ACCURACY OF DIFFERENCE APPROXIMATIONS TO PLANE DIRICHLET PROBLEMS WITH PIECEWISE ANALYTIC BOUNDARY VALUES ${ }^{1}$
}

\author{
BY \\ WOLFGANG R. WASOW \\ University of California at Los Angeles
}

1. Introduction. The standard method for appraising the difference between the exact solution $u$ of a problem involving a partial differential equation and the solution $U$ of an approximating finite difference problem is based on the expansion of $u$ by Taylor's formula up to terms of third or higher degree. The partial derivatives of $u$ that enter the argument in this manner are unbounded near the boundary $C$ of the region $R$ where the problem is to be solved, unless $C$ and the prescribed boundary values are very smooth.

In computational practice the boundary and the prescribed boundary values are almost always piecewise analytic. At the corners of $C$ and at the points of $C$, where the boundary values have a jump in the first or second derivative the higher derivatives of $u$ become usually unbounded. Therefore there prevails the unsatisfactory situation that most known appraisals of the truncation error $U-u$ in the numerical solution of boundary value problems are based on assumptions which are hardly ever satisfied in computational practice.

The present note is meant as a first step to overcome this difficulty. It will be shown that for the simplest finite difference approximation to Dirichlet's problem for Laplace's equation the order of magnitude of the truncation error is not affected by jumps in the first derivative of the boundary function. Of course, this is true only outside the immediate neighborhood of those discontinuities.

It is frequently contended that, as the data of a mathematical problem of physical origin are by their very nature only approximate, the disconitinuities in the derivatives of boundary values can be safely ignored in problems of this nature. However, there are many problems where these discontinuities are a very accurate model of physical reality, while their elimination by a smooth connecting arc would change either $u$ or $U$ in a manner that cannot be guaranteed to be small without a further investigation like the one given in this paper.

It is hoped that the method of this paper can be extended to more refined finite difference approximations and to other differential problems as well as to problems where the boundary $C$ has corners.

2. Green's function for the difference equation. Let $C$ be a simple closed analytic curve on which is defined a continuous function $f(s)$ that is piecewise analytic. By this statement we mean that $C$ can be described by two analytic functions $x=x(s), y=y(s)$ of period $l$, regular for real $s$, and that there exists a finite number of values of $s$, say $0 \leq s_{1}<s_{2} \cdots<s_{n}<l$, such that $f(s)$ is continuous, periodic with period $l$, and regular analytic in every one of the intervals $s_{\nu} \leq s \leq s_{v+1}, \nu=1, \cdots, n$, and $s_{n} \leq s \leq s_{1}+l$. Furthermore, we require that $(d x / d s)^{2}+(d y / d s)^{2} \neq 0$.

Denote the interior of $C$ by $R$. Then there exists a unique function $u(x, y)$ for which

$$
\Delta u=0 \quad \text { in } R, \quad u=f \quad \text { on } C .
$$

'Received January 23, 1956. This paper was sponsored by the Office of Ordnance Research and the Office of Naval Research. Reproduction in whole or in part is permitted for any purpose of the United States Government. 
The function $u(x, y)$ is regular analytic on $C$, except possibly at the points $S_{\nu}(\nu=1$, $\cdots, n$ ) of $C$ that correspond to the parameter values $s_{\text {, }}$.

We denote by $\Delta_{h}$ the finite difference operator defined by

$$
\Delta_{h} U=h^{-2}[U(x+h, y)+U(x-h, y)+U(x, y+h)+U(x, y-h)-4 U(x, y)] .
$$

Let $R_{h}$ be the set consisting of all net points $P$ in $R$ such that the four nearest neighbors of $P$ in the grid lie in $R+C$, and denote by $C_{h}$ the set of net points in $R+C$ which do not belong to $R_{h}$. On $C_{h}$ we prescribe a function $f_{h}=f_{h}(P)$ whose value at a point $P$ of $C_{h}$ is equal to $f(s)$ at some point $P^{\prime}$ of $C$ for which $\overline{P P^{\prime}}<h$.

The problem (1) is to be approximated by

$$
\Delta_{h} U=0 \quad \text { in } R_{h}, \quad U=f_{h} \quad \text { on } C_{h} .
$$

It is known that the truncation error $v=U-u$ is $O(h)$ if $\partial^{3} u / \partial x^{3}$ and $\partial^{3} u / \partial y^{3}$ are uniformly bounded in $R$. This was first proved by Gerschgorin in [1] by means of the maximum principle for the operator $\Delta_{h}$, which states that a function $U$ for which $\Delta_{h} U=0$ in a set of gridpoints cannot have an extreme value in this set unless it is a constant. Since the third derivatives of $u$ diverge near the points $S$, of $C$, a stronger tool than the maximum principle is now needed. Our study of the truncation error is based on the asymptotic properties, for $h \rightarrow 0$, of Green's function for the operator $\Delta_{h}$ in $R_{h}$.

For the definition of Green's function we consider the problem

$$
\Delta_{h} V=\varphi(x, y) \quad \text { in } R_{h}, \quad V=0 \quad \text { on } C .
$$

Its solution is clearly a linear combination of the values of $\varphi(x, y)$ at the points of $R_{h}$, so that we may write

$$
V(P)=h^{2} \sum_{Q \in R_{\star}} G_{h}(P, Q) \varphi(Q) .
$$

Here we have written $\varphi(Q)$ for $\varphi(\xi, \eta)$, etc. The factor $h^{2}$ has been extracted in anticipation of a comparison with Green's function for the differential problem. $G_{h}(P, Q)$ will be called Green's function for the operator $\Delta_{h}$ in $R_{h}$. If (3) is interpreted as a system of $N$ linear algebraic equations for the values of $V(P)$ at the $N$ points of $R_{h}$, the $N^{2}$ values of $h^{2} G_{h}(P, Q)$ form a matrix which is the inverse of the coefficient matrix in the system for $V(P)$. Since the latter matrix is symmetric, so is $G_{h}(P, Q)$ :

$$
G_{h}(P, Q)=G_{h}(Q, P) \text {. }
$$

By applying (3) and (4) to the particular function $\varphi(Q)=\delta\left(Q, Q^{\prime}\right)$, where

$$
\delta\left(Q, Q^{\prime}\right)= \begin{cases}0, & Q \neq Q^{\prime} \\ 1, & Q=Q^{\prime}\end{cases}
$$

it is seen that $G_{h}(P, Q)$ is the solution of the problem

$$
\begin{array}{rlrl}
\Delta_{h, P} G_{h}(P, Q) & =h^{-2} \delta(P, Q), & P \varepsilon R_{h}, \\
G_{h}(P, Q) & =0 \quad, \quad P \varepsilon C_{h} .
\end{array}
$$

The subscript $P$ in the symbol $\Delta_{h . P}$ means that the operator is to be applied with respect to the variable $P$. The function $G_{h}(P, Q)$ is non-negative in $R_{h}$. For, $G_{h}(P, Q)$ cannot 
be a constant, because of (7). If it is negative anywhere in $R_{h}$, there must be a point $P=P_{0}$ in $R_{k}$ where $G_{k}(P, Q)$, as function of $P$ for fixed $Q$, has a negative minimum, while $G_{h}\left(P_{i}, Q\right)>G_{h}\left(P_{0}, Q\right)$ for at least one of the four nearest neighbors $P_{1},(j=1, \cdots, 4)$, of $P_{0}$ in the grid. However, the difference equation in (7) implies that

$$
G_{h}\left(P_{0}, Q\right) \geq 1 \sum_{i=1}^{4} G\left(P_{i}, Q\right),
$$

and therefore $G_{h}\left(P_{0}, Q\right)$ must also exceed at least one of the four values $G_{h}\left(P_{i}, Q\right)$, which is a contradiction to the minimum property of $P_{0}$.

The asymptotic study of $G_{h}(P, Q)$ is based on certain results contained in [2]. It will be convenient to write Sin and Cos instead of the usual symbols sinh, cosh for the hyperbolic function in order to avoid confusion with the trigonometric functions of the mesh length $h$. We shall be concerned with the function

$$
\chi(\sigma, \tau)=\frac{2}{\pi} \int_{0}^{\pi} \frac{1-\cos (\tau \lambda) e^{-|\sigma| \mu}}{\sinh \mu} d \lambda,
$$

where $\mu$ is the function of $\lambda$ defined, for $0<\lambda<\pi$, by the equation

$$
\cos \lambda+\cosh \mu=2
$$

and the condition $\lim \mu / \lambda=1$. It was shown by McCrea and Whipple [2] that $\chi(\sigma, \tau)$ has"the following properties.

1. $\chi(\sigma, \tau)=\chi(\tau, \sigma)$,

2. $\chi(0,0)=0$,

3. $\chi(\sigma+1, \tau)+\chi(\sigma-1, \tau)+\chi(\sigma, \tau+1)+\chi(\sigma, \tau-1)-4 \chi(\sigma, \tau)$

$$
= \begin{cases}0, & \tau^{2}+\sigma^{2} \neq 0 \\ 4, & \sigma=\tau=0,\end{cases}
$$

4. $\chi(\sigma, \tau)=\frac{1}{\pi} \log \left(\sigma^{2}+\tau^{2}\right)+c+0\left(\sigma^{-2}\right), \quad$ uniformly in $\tau$,

as $\sigma \rightarrow \infty$. Here, $c=(1 / \pi)(\log 8+2 \gamma)$, and $\gamma$ is Euler's constant.

Actually, McCrea and Whipple give only $0\left(\sigma^{-1}\right)$ as the order of the remainder terms, but their own calculations show that the stronger result is true. For reasons of symmetry we replace the error term by $0\left[1 /\left(\sigma^{2}+\tau^{2}\right)\right]$, which is permissible in view of property 1 .

It follows, then, that the function

$$
\gamma_{h}(x, y)=\frac{1}{4} \chi\left(\frac{x}{h}, \frac{y}{h}\right)+\frac{1}{2 \pi} \log h-c
$$

has the properties

$$
\begin{aligned}
& 1^{\prime} . \gamma_{h}(x, y)=\gamma_{h}(y, x), \\
& \text { 2'. }^{\prime} \gamma_{h}(0,0)=\frac{1}{2 \pi} \log h-c,
\end{aligned}
$$


$3^{\prime} . \quad \Delta_{h} \gamma_{h}(x, y)= \begin{cases}0, & x^{2}+y^{2} \neq 0 \\ h^{-2}, & x=y=0,\end{cases}$

4'. $\gamma_{h}(x, y)=\frac{1}{2 \pi} \log \left(x^{2}+y^{2}\right)^{1 / 2}+0\left(\frac{h^{2}}{x^{2}+y^{2}}\right)$.

The function $-\gamma_{h}(x, y)$ is a discrete analog of a fundamental solution for Laplace's equation. We now introduce the function

$$
H_{h}(P, Q)=-\gamma_{h}(x-\xi, y-\eta),
$$

where $(x, y),(\xi, \eta)$ are the coordinates of the points $P, Q$, respectively. Then we have, by property $3^{\prime}$,

$$
\Delta_{h, P} H_{h}(P, Q)=h^{-2} \delta(P, Q) .
$$

In order to compare the asymptotic behavior of $H_{h}(P, Q)$ with that of $G_{h}(P, Q)$ we introduce the difference

$$
e_{h}(P, Q)=G_{h}(P, Q)-H_{h}(P, Q),
$$

which, because of (7) and (9), satisfies the difference equation

$$
\Delta_{h, p} e_{h}(P, Q)=0, \quad P \varepsilon R_{h}
$$

and the boundary condition

$$
e_{h}(P, Q)=-H_{h}(P, Q), \quad P_{\varepsilon} C_{h} .
$$

We shall show that

$$
e_{h}(P, Q)=\psi(P, Q)+O(h), \quad \text { for } P \varepsilon R_{h}, \quad Q \neq P, \quad \text { and } Q \text { not on } C,
$$

where $\psi(P, Q)$ is, for $P \varepsilon R$, the harmonic function of $P$ with boundary values

$$
\psi(P, Q)=\frac{1}{2 \pi} \log \overline{P Q}, \quad P_{\varepsilon} C .
$$

To this end we expand $\Delta_{h . P} \psi(P, Q)$ by Taylor's formula and use the fact that $\psi(P, Q)$ is, for $Q$ not on $C$, a harmonic function of $P$ in the closed domain $R+C$, thanks to the analyticity of the curve $C$ (see [5], p. 187). Then we obtain

$$
\Delta_{h, P} \psi(P, Q)=O\left(h^{2}\right), \quad P \varepsilon R_{h}, \quad Q \text { not on } C .
$$

Also, by property $4^{\prime}$ and formulas (8) and (14),

$$
\psi(P, Q)=-H_{h}(P, Q)+O(h), \quad P_{\varepsilon} C_{h}, \quad P \neq Q .
$$

The last two formulas are valid uniformly in $Q$, if $Q$ is bounded away from $C$ by a positive distance independent of $h$ and if $\overline{P Q} \geq a h^{1 / 2}$, where $a$ is a positive constant independent of $h$. If we subtract (15) and (16) from (11) and (12) we find

$$
\begin{array}{rll}
\Delta_{h, P}\left[e_{h}(P, Q)-\psi(P, Q)\right]=O\left(h^{2}\right), & P \varepsilon R_{h}, & Q \neq P, \\
e_{h}(P, Q)-\psi(P, Q)=O(h), & P \varepsilon C_{h}, & P \neq Q .
\end{array}
$$

It is well known, and easy to prove by means of the maximum principle for the operator $\Delta_{h}$, (see [1]) that the Eqs. (17), (18) imply indeed (13). 
Finally, we replace $e_{h}(P, Q)$ by its definition (10), and use once more property $4^{\prime}$. Then we see from (13) that

$$
G_{h}(P, Q)=-\frac{1}{2 \pi} \log \overline{P Q}+\psi(P, Q)+O(h), \quad P \varepsilon R_{h}, \quad Q \neq P, \quad Q \text { not on } C .
$$

The first two terms in the right member together constitute Green's function in $R$ for Laplace's operator $\Delta$. Hence,

$$
G_{h}(P, Q)=G(P, Q)+O(h), \quad \text { for } P \varepsilon R_{h}, \quad Q \neq P, \quad Q \text { not on } C .
$$

We repeat that this relation is uniformly valid, if $Q$ is bounded away from $C$ by a positive distance independent of $h$, and if $\overline{P Q} \geq a h^{1 / 2}$.

3. The behavior of harmonic functions near corners of the boundary values. We consider first the special case that $C$ is the unit circle and that $u(x, y)=u_{m}(x, y)$ is a function from the sequence defined recursively by

$$
\begin{gathered}
u_{m}(x, y)=\operatorname{Re} F_{m}(z), \quad z=x+i y, \\
F_{0}(z)=i \log (1-z), \quad F_{m}(z)=-i \int_{1}^{z} F_{m-1}(t) d t, \quad t \varepsilon R+C .
\end{gathered}
$$

If

$$
1-z=\rho e^{i \alpha},
$$

we take the branch of $\log (1-z)$ defined by $|\alpha| \leq \pi / 2$. Then $F_{0}(z)$ is regular analytic in $R+C$ except at $z=1$, and

$$
F_{0}(z)=u_{0}(x, y)+i v_{0}(x, y)=-\alpha+i \log \rho .
$$

On the boundary,

$$
u_{0}(x, y)=f_{0}(\theta)=\frac{\pi-\theta}{2}, \quad 0<\theta<2 \pi .
$$

Hence, $f_{0}(\theta)$ is continuous except for a jump of size $\pi$ at $\theta=0$. For $m>0$ the functions $u_{m}(x, y)$ and $v_{m}(x, y)=\operatorname{Im} F_{m}(z)$ are continuous in $R+C$. On $C$ we have $z=e^{i \theta}$ and therefore

$$
\frac{d^{\nu}}{d \theta^{\nu}} F_{m}(z)=F_{m-\nu}(z) e^{i \nu \theta}+\cdots, \quad z \varepsilon C,
$$

i.e. the boundary values $f_{m}(\theta)$ of $u_{m}(x, y)$ have the derivatives

$$
f_{m}^{(v)}(\theta)=f_{m-\nu}(\theta) \cos \nu \theta-v_{m-\nu}(x, y) \sin \nu \theta+\cdots, \quad(x, y) \varepsilon C,
$$

where the dots indicate terms involving higher subscripts. This proves the continuity of $f_{m}^{(\nu)}(\theta)$ for $\nu<m$. For $\nu=m$, the first righthand term has a jump of size $(-1)^{m} \pi$ at $\theta=0$. The second term equals $\log \rho \sin \nu \theta$, which has the limit 0 at $\theta=0$. All the other terms are continuous, so that the jump of $(-1)^{m} \pi$ characterizes the behavior of $f_{m}^{(m)}(\theta)$ at $\theta=0$.

The partial derivatives of $u_{m}(x, y)$ of order $\nu<m$ are continuous in $R+C$. This follows directly from the definition of $u_{m}(x, y)$ in (20) and the Cauchy-Riemann equations. The $m$ th derivatives are multiples of $u_{0}(x, y)$ or $v_{0}(x, y)$ and are therefore $0(\log \rho)$, 
at worst. If $\nu>m$, the derivatives are - except for numerical factors-the real or imaginary part of $d^{\nu-m} / d z^{\nu-m} \log (1-z)$. Their order of magnitude is, thus, $0\left(\rho^{p-m}\right)$.

Now assume that the boundary function $f(s)=f(\theta)$ of $u(x, y)$ is regular analytic on the unit circle $C$ except at $\theta=0$, (i.e. $z=1$ ), where it has a jump of size $K$ in the $p$ th derivative, while the derivatives of lower order are continuous. The function $f(\theta)-$ $(-1)^{p}(K / \pi) f_{p}(\theta)$ has then continuous derivatives on $C$ up to order $p$ inclusive. If it has a discontinuity in the $(p+1)$ st derivative, this can also be eliminated by addition of a suitable multiple of $f_{p+1}(\theta)$, and so forth. Hence, there exists a linear combination

$$
f^{*}(\theta)=f(\theta)+\alpha_{0} f_{p}(\theta)+\alpha_{1} f_{p+1}(\theta)+\cdots+\alpha_{q} f_{p+\theta}(\theta)
$$

with continuous derivatives of order $p+q$ and, at worst, a jump in the $(p+q+1)$ st derivative. The harmonic function with boundary values $f^{*}(\theta)$ on $C$ possesses then continuous derivatives up to order $p+q$, inclusive in $R+C$ (see [3], p. 243]. Therefore

$$
\begin{aligned}
\frac{\partial^{p+a} u}{\partial x^{p+a}} & =O\left[\alpha_{0} \frac{\partial u_{p}^{p+a}}{\partial x^{p+q}}+\cdots+\alpha_{a} \frac{\partial^{p+a} u_{p+a}}{\partial x^{p+a}}\right] \\
& =O\left[\frac{\partial u_{p}^{p+q}}{\partial x^{p+a}}\right]= \begin{cases}O(\log \rho), & q=0 \\
O\left(\rho^{-q}\right), & q>0\end{cases}
\end{aligned}
$$

and, similarly,

$$
\frac{\partial^{p+q} u}{\partial y \partial x^{p+q-1}}= \begin{cases}O(\log \rho), & q=0, \\ O\left(\rho^{-q}\right), & q>0 .\end{cases}
$$

A similar discontinuity of a derivative of $f(\theta)$ at any other point $\theta=\theta_{0}$ on the unit circle $C$ can be analyzed by means of auxiliary harmonic functions obtained from $u_{m}(x, y)$ by a rotation through the angle $\theta_{0}$. If more than one point with discontinuities in the derivatives of $f(\theta)$ occur they can be handled simultaneously by adding a sum of appropriate compensating harmonic functions. Finally, if $C$ is not the unit circle $R$ can be changed into the interior of the unit circle by a conformal mapping. The mapping function and its inverse transform harmonic functions in $R$ into harmonic functions inside the unit circle, and conversely. Since $C$ is analytic the mapping function is analytic in $C+R$ and hecne the transformed boundary function is harmonic at all points of the unit circle except those corresponding to the points $S_{i}, j=1, \cdots, n$ on $C$. Furthermore, the orders of magnitude of the harmonic function in $R$ near the points $S_{i}$ are the same as those of the image function in the unit circle. Hence, we have proved that the solution $u(x, y)$ of problem (1) is harmonic in $R+C$ except at the boundary points $S_{i}$, where $f(\theta)$ is not analytic. At those points the order of magnitude of the derivatives for approach in $R+C$ is determined by the formulas (21) and (22).

4. The truncation error for Dirichlet's problem. The truncation error $v=U-u$ for the approximate solution of problem (1) by means of problem (2) is the solution of the problem

$$
\begin{aligned}
& \Delta_{h} v=-\Delta_{h} u, \quad \text { in } R_{h}, \\
& v=f_{h}-u, \quad \text { on } C_{h} \text {. }
\end{aligned}
$$


By Taylor's formula we have

$$
\begin{aligned}
\Delta_{h} u(x, y)=\frac{h}{6}\left[\frac{\partial^{3}}{\partial x^{3}} u(x+\theta h, y)-\frac{\partial^{3}}{\partial x^{3}} u(x-\theta h, y)+\frac{\partial^{3}}{\partial y^{3}} u(x, y+\theta h)\right. & \\
& -\frac{\partial^{3}}{\partial y^{3}} u(x, y-\theta h]
\end{aligned}
$$

with $0<\theta<1$. We know from the preceding section that $\partial^{8} / \partial x^{3} u(Q)$ and $\partial^{3} / \partial y^{3} u(Q)$ are $0\left(\widehat{Q S}_{i}^{-2}\right)$ near $S_{i}$. For simplicity we consider only the case that there is no more than one singular point $S_{i}=S$ on $C$, and set $\overline{Q S}=\rho$. The extension to a finite number of such points is trivial.

From now on we must subject the choice of grids to the important restriction that the distance of the points $S_{i}$ on $C$ from the nearest grid line be at least bh, $0<b<1$, where $b$ is independent of $h$. Then the right member of (25) does not exceed $K_{1} h_{\rho}^{-2}$ in $R$, where $K_{1}$ is a constant depending on $f(s)$ and on $b$, not on $h$, i.e.

$$
\left|\Delta_{h} u\right| \leq K_{1} h_{\rho}{ }^{-2} \text {. }
$$

In order to arrive at an appraisal of $v$ we represent $v$ as the sum $v=v_{1}+v_{2}$ of the solutions of the problems

$$
\begin{aligned}
\Delta_{h} v_{1} & =-\Delta_{h} u, & & \text { in } R_{h} \\
v_{1} & =0, & & \text { on } C_{h}
\end{aligned}
$$

and

$$
\begin{aligned}
\Delta_{h} v_{2}=0, & \text { in } R_{h}, \\
v_{2}=f_{h}-u, & \text { on } C_{h} .
\end{aligned}
$$

Discussing $v_{1}$ first we represent this function in the form (4) and make use of (26), obtaining

$$
\left|v_{1}(P)\right| \leq h K_{1} \sum_{Q \in R_{h}} h^{2} G_{h}(P, Q) \rho^{-2} .
$$

If we extend the definition of $G_{h}(P, Q)$ in an appropriate manner from the points of $R_{h}+C_{h}$ to all points of $R+C$, the sum in the right member can be replaced by an integral. To this end we associate with every square of the grid that lies entirely in $R+C$ the value of $G_{h}(P, Q)$ as function of $Q$, at its lower left vertex. At the remaining points of $R$ we define $G_{h}(P, Q)$ as being zero. Then

$$
\sum_{Q \in R_{h}} h^{2} G_{h}(P, Q) \rho^{-2}=\iint_{R} G_{h}(P, Q) \rho^{-2} d Q+O(h) .
$$

Since $G_{h}(P, Q)$ is symmetric in $P$ and $Q$, we can appraise the integral above by means of the asymptotic formula (19), provided $P$ is restricted to some proper closed subdomain $R^{\prime}$ of $R$. Let

$$
R=R_{1}+R_{2}+R_{3},
$$

where $R_{1}$ is the circular region $\overline{P Q}<a h^{1 / 2}$ about $P$, the domain $R_{2}$ is closed and satisfies $R^{\prime} \subset R_{1}+R_{2} \subset R$, and, finally, $R_{3}=R-R_{1}-R_{2}$. 
We show first that

$$
\iint_{R_{1}} G_{h}(P, Q) \rho^{-2} d Q=O(h \log h) .
$$

To see this we observe that the function $\left|e_{h}(P, Q)\right|$ assumes its maximum on $C_{h}$, because of (11). In view of (8), (12) and the definition of $\gamma_{h}(x, y)$ it follows that $\left|e_{h}(P, Q)\right|$ remains uniformly bounded, for $Q_{\varepsilon} R^{\prime}$, as $h \rightarrow 0$. Since $H_{\Lambda}(P, Q)=O(\log h)$, in consequence of property $2^{\prime}$, we see from (10) that

$$
G_{h}(P, Q)=O(\log h),
$$

if $Q \varepsilon R^{\prime}$ or if $P \varepsilon R^{\prime}$ (the latter because of the symmetry of Green's function). This proves (31).

Furthermore

$$
\iint_{R_{2}} G_{h}(P, Q) \rho^{-2} d Q=\iint_{R_{I}} G(P, Q) \rho^{-2} d Q+\iint_{R_{z}} O(h) \rho^{-2} d Q,
$$

by (19). The first integral in the right member exists, because $\rho^{-1}$ is bounded in $R_{2}$, and the last integral is $O(h)$. Thus

$$
\iint_{R_{2}} G_{h}(P, Q) \rho^{-2} d Q=O(1) .
$$

In the remaining integral $\iint_{R_{2}} G_{h}(P, Q) \rho^{-2} d Q$ we make use of the fact that $G_{h}(P, Q)$ is, by definition, zero whenever $Q$ is a point of a gridsquare that does not lie entirely in $R+C$. The point $S$ lies in such a square; in fact, it has at least the distance $b h$ from the edges of this square by virtue of the hypothesis introduced in the paragraph after formula (25). Therefore, $R_{3}$ may be replaced in the integration by a subregion $R_{3}^{*}$ that has at least the distance $b h$ from $S$. Using again (19), it follows that

$$
\iint_{R_{*}} G_{h}(P, Q) \rho^{-2} d Q=\iint_{R_{0^{*}}} G(P, Q) \rho^{-2} d Q+\iint_{R_{2^{*}}} O(h) \rho^{-2} d Q .
$$

The first integral in the right member is bounded, because $G(P, Q)$ vanishes on the boundary and is there continuously differentiable so that

$$
G(P, Q)<K_{2} \rho, \quad P \varepsilon R^{\prime}, \quad Q \varepsilon R_{3} .
$$

The last integral in the right member does not exceed

$$
b^{-1} \iint_{R, \bullet}|O(h)| h^{-1} \rho^{-1} d Q,
$$

which is bounded. Therefore

$$
\iint_{R_{3}} G_{h}(P, Q) \rho^{-2} d Q=O(1) .
$$

If (31), (34) and (35) are inserted into (30), the inequality (29) is seen to imply that

$$
v_{1}(P)=O(h), \quad P \varepsilon R^{\prime} .
$$


We now turn to the appraisal of $v_{2}$. In order to make our analysis of Green's function available for this problem, we use Green's formula for the operator $\Delta_{h}$, which can be written

$$
h^{2} \sum_{R_{\Lambda}}\left(V \Delta_{h} U-U \Delta_{h} V\right)+h \sum_{C_{\Lambda}}\left(V \Gamma_{h} U-U \Gamma_{h} V\right)=0,
$$

(see [4], p. 36). Here $U$ and $V$ may be any two functions in the grid, and the operator $\Gamma_{h} U$ is defined as follows: Let $Q$ be a point of $C_{h}$ and denote by $Q_{i},(j=1, \cdots \nu \leq 3)$ the gridpoints in $R_{k}$ at distance $h$ from $Q$, then

$$
\Gamma_{h} U(Q)=h^{-1}\left[U\left(Q_{1}\right)+\cdots+U\left(Q_{v}\right)-\nu U(Q)\right] .
$$

We apply (37) with $V=v_{2}(Q), U=G_{h}(P, Q)$ and find, using (7) and (28), that

$$
v_{2}(P)=-h \sum_{0, C_{h}} \Gamma_{h, Q}\left[G_{h}(P, Q)\right]\left[f_{h}(Q)-u(Q)\right] .
$$

Now, by definition, $f_{\wedge}(Q)=f\left(Q^{\prime}\right)$ where $Q^{\prime}$ lies on $C$, and $\overline{Q Q^{\prime}}<h$. In analogy with the hypothesis introduced after formula (25), we have to restrict the choice of $Q^{\prime}$ somewhat by requiring that the distance from $S$ to the points of the segment $Q Q^{\prime}$ be at least bh uniformly for $Q$ on $C_{n}$. By the theorem of the mean

$$
f_{h}(Q)-u(Q)=f\left(Q^{\prime}\right)-u(Q)=\frac{\partial}{\partial \sigma} u\left(Q^{\prime \prime}\right) \cdot \overline{Q Q^{\prime}},
$$

where $\partial / \partial \sigma$ indicates differentiation in the direction from $Q^{\prime}$ to $Q$ and $Q^{\prime \prime}$ is a point between $Q$ and $Q^{\prime}$. We proved in Sec. 3 that the first derivative of $u$ at the point $Q^{\prime \prime}$ in any direction is $0\left(\log \rho^{\prime \prime}\right)$, the letter $\rho^{\prime \prime}$ designating the distance $\overline{S Q^{\prime \prime}}$. With the help of our restriction on $Q^{\prime}$ we shall show first that

$$
\left|f_{h}(Q)-u(Q)\right| \leq K_{3} h(|\log \rho|+1),
$$

$K_{\mathrm{s}}$ being independent of $h$ and $\rho$. By assumption, $\rho \geq b h$ and ${\overline{Q Q^{\prime \prime}}}^{\prime \prime} \overline{Q Q^{\prime}} \leq h$, whence $\overline{Q Q}^{\prime \prime} \leq \rho / b$. Therefore, $\rho^{\prime \prime} \leq \rho+\overline{Q Q}^{\prime \prime} \leq \rho(1+1 / b)$. Using our other assumption that $\rho^{\prime \prime} \geq b h$ we show analogously that $\rho \leq \rho^{\prime \prime}+Q Q^{\prime \prime}<\rho^{\prime \prime}(1+1 / b)$. The ensuing double inequality

$$
\log \rho-\log \left(1+\frac{1}{b}\right) \leq \log \rho^{\prime \prime} \leq \log \rho+\log \left(1+\frac{1}{b}\right)
$$

implies that

$$
\begin{aligned}
\left|\log \rho^{\prime \prime}\right| & \leq \max \left\{\left|\log \rho+\log \left(1+\frac{1}{b}\right)\right|,\left|\log \rho-\log \left(1+\frac{1}{b}\right)\right|\right\} \\
& \leq|\log \rho|+\log \left(1+\frac{1}{b}\right),
\end{aligned}
$$

and, therefore,

$$
\left|\frac{\partial}{\partial \sigma} u\left(Q^{\prime \prime}\right) \cdot \overline{Q Q^{\prime}}\right|=O\left(h \log \rho^{\prime \prime}\right) \leq K_{3} h(|\log \rho|+1) .
$$

On $C_{h}$ the relation (19) can be used, if $P \varepsilon R^{\prime}$, and this implies that $G_{h}(P, Q)$ is $O(h)$, for $Q \& C_{A}$. Using (38) and (40) we obtain then from (39) the inequality 


$$
\left|v_{2}(P)\right| \leq K_{4} h \sum_{c_{\star}} h(|\log \rho|+1), \quad\left(K_{4} \text { a constant }\right) .
$$

It is plausible that

$$
\sum_{C_{A}} h(|\log \rho|+1)=O(1) .
$$

The simple proof of this statement will be postponed to the end of this section. On the basis of (41) and (42) we have

$$
\left|v_{2}(P)\right|=O(h), \quad P \varepsilon R^{\prime} .
$$

This relation, together with (36) completes the proof of our main result, which we now state as a formal theorem.

Theorem: The truncation error $v(P)=U(P)-u(P)$ corresponding to the approximate solution of problem (1) by means of the equations (2) is of order $O(h)$, provided the following conditions are satisfied:

(a) the boundary $C$ is a simple closed analytic curve;

(b) the boundary function $f(s)$ is continuous and piecewise analytic;

(c) the distance from the singularities of $f(s)$ to the nearest point on a grid line is not less than $b h,(0<b<1$, independent of $h)$.

(d) If $f_{A}(Q)=f\left(Q^{\prime}\right), Q^{\prime} \varepsilon C$, then the distance of the singularities of $f(s)$ from the segment $Q Q^{\prime}$ is not less than $b h$.

The truncation error $v(P)$ has the order $O(h)$ uniformly in every closed subdomain of $R$.

Proof of formula (42). We show first that the total number $M$ of the gridpoints in $C_{\mathrm{a}}$ is $O\left(h^{-1}\right)$. The analytic curve $C$ possesses only a finite number of points where either $d x / d s$ or $d y / d s$ vanishes. These points divide $C$ into a finite number of arcs each of which does not intersect the same grid line twice. Hence, if $C^{0}$ is one such arc and if the lengths of its projections on the axes are $L_{x}$ and $L_{y}$, respectively, then the arc $C^{0}$ possesses at most $\left(L_{z}+L_{y}+2\right) h^{-1}$ points of intersection with lines of the grid. It follows that the total number of intersections of $C$ with lines of the grid is $O\left(h^{-1}\right)$. Now, every point of $C_{\mathrm{A}}$ is an end point of a mesh side of length $h$ that has a point in common with $C_{i}$. Hence, $M=O\left(h^{-1}\right)$, as claimed, say,

$$
M \leq L h^{-1} \text {. }
$$

For sufficiently small $h$ no closed segment of length $h$ joining two gridpoints will have more than one point in common with $C$. Then exactly one of the two end points of such a segment belongs to $C_{\mathrm{h}}$. We now measure the arc length $s$ on $C$ from $S$ and order the points $P_{r}$ of $C_{\mathrm{A}}$ in such a way that $r_{1}<r_{2}$ if and only if $P_{r_{1}}$ is an end point of a segment that meets $C$ at a point with smaller value of $s$ than any grid segment ending at $P_{r}$. . Then $\overline{S P}_{\mathrm{r}} \leq 2^{1 / 2} r h$ and, therefore,

$$
\begin{aligned}
\sum_{c_{\Delta}} h(|\log \rho|+1) & \leq \sum_{r=1}^{M} h\left(\left|\log 2^{1 / 2} r h\right|+1\right) \\
& \leq \int_{0}^{L+\hbar}\left(\left|\log 2^{1 / 2} t\right|+1\right) d t \leq K_{5}, \quad \text { (K } \text { a constant), }
\end{aligned}
$$

which proves (42). 


\section{BIBLIOGRAPHY}

1. S. Gerschgorin, Fehlerabschätzung für das Differenzenverfahren zur Lösung partieller Differentialgleichungen, Z. angew. Math. Mech. 10, 373-382 (1930)

2. W. H. McCrea and F. J. W. Whipple, Random paths in two and three dimensions, Proc. Roy. Soc. Edinburgh 60, 281-298 (1939-40)

3. L. Lichtenstein, Neuere Entwicklungen der Potentialtheorie. Konforme Abbildung, Enzyk. der math. Wiss. II C 3, vol. 2, pt. 3, Bd. 1, 177-377, Leipzig (1918)

4. R. Courant, K. Friedrichs and $H$. Lewy, Uber die partiellen Differenzengleichungen der mathematischen Physik, Math. Ann. 100, 32-74 (1928)

5. Z. Nehari, Conformal mapping, New York, 1952. 